\title{
Shallow Whole Genome Sequencing for the Assembly of Complete Chloroplast Genome Sequence of Arachis hypogaea L.
}

\author{
Sudheesh K. Prabhudas, Sowjanya Prayaga, Parani Madasamy and \\ Purushothaman Natarajan * \\ Department of Genetic Engineering, SRM University, Kattankulathur, India
}

Keywords: complete chloroplast genome, Arachis hypogaea, peanut, groundnut, de novo assembly, illumina

\section{OPEN ACCESS}

Edited by:

Thiago Motta Venancio, Universidade Estadual do Norte

Fluminense, Brazil

Reviewed by:

Rafael Dias Mesquita,

Federal University of Rio de Janeiro,

Brazil

Swarup Kumar Parida,

National Institute of Plant Genome

Research, India

*Correspondence:

Purushothaman Natarajan purushothaman.n@ktr.srmuniv.ac.in

Specialty section:

This article was submitted to Bioinformatics and Computational

Biology,

a section of the journal

Frontiers in Plant Science

Received: 16 June 2016

Accepted: 12 July 2016

Published: 27 July 2016

Citation:

Prabhudas SK, Prayaga S, Madasamy P and Natarajan P (2016) Shallow Whole Genome Sequencing

for the Assembly of Complete

Chloroplast Genome Sequence of

Arachis hypogaea L.

Front. Plant Sci. 7:1106. doi: 10.3389/fp/s.2016.01106

\section{INTRODUCTION}

The chloroplast (CP) is a plant organelle originated from cyanobacteria through symbiosis and had become an important component of the plant cell. It is the reaction center for the photosynthesis and also for several steps in the biosynthetic pathways of fatty acids, vitamins, pigments and amino acids. The CP genome is highly conserved in land plants (Raubeson and Jansen, 2005). The CP genome is circular and exhibits a quadripartite genome structure consisting of a large single copy region (LSC) and a small single copy region (SSC), separated by a pair of inverted repeats (IRs) with a few exceptions where loss of an IR or the SSC was observed. The size of the $\mathrm{CP}$ genome varies from 19 to $217 \mathrm{~Kb}$ in land plants, and the IRs are usually $20-26 \mathrm{~kb}$ in length (http://www.ncbi.nlm.nih.gov/genome/organelle/). Lack of recombination makes the CP genome an ideal target for phylogenetic studies (Ravi et al., 2008; Wu and Ge, 2012).

Arachis hypogaea $\mathrm{L}$. also known as groundnut is an herbaceous plant belonging to the Fabaceae family. It has an allotetraploid genome (AABB; $2 \mathrm{n}=4 \mathrm{x}=40$ ) with a size of about $2.8 \mathrm{~Gb}$. There have been many speculations regarding the ancestors of $\mathrm{A}$ and $\mathrm{B}$ subgenomes of $A$. hypogaea and proved to have originated through a hybridization event between Arachis ipaensis L. (B subgenome) and Arachis duranensis L. (A subgenome) (Kochert et al., 1996; David et al., 2016). It is one of the major edible oilseed crops in the world, and India is the second largest producer accounting for about $15 \%$ of the world production (FAOSTAT, 2015). Kernels of A. hypogaea L. contains 43$50 \%$ oil and $23-26 \%$ proteins. The oil comprises majorly of palmitic acid (16:0), stearic acid (18:0), oleic acid (18:1), linoleic acid (18:2), arachidic acid (20:0), eicosenoic acid (20:1), behemic acid (22:0), and lignoseric acid (24:0) along with trace amounts of palmitoleic acid (16:1). The mono and poly-unsaturated fatty acids, oleic acid and linoleic acid constitute about $75 \%$ of the total oil content (Shiv, 1982). Many attempts have successfully been made to improve the crop yield, drought resistance, disease resistance and other characteristics of A. hypogaea L. using classical breeding as well as genetic engineering using nuclear transformation. Chloroplast transformation by homologous recombination for producing transgenic plants is also possible due to the presence of candidate loci on the $\mathrm{CP}$ genome. Additionally, Genetic engineering of chloroplast genome when compared to nuclear transformation is environment-friendly; it minimizes the pleiotropic effects along with containment of the foreign genes (Daniell et al., 2005). Hence, the availability of the complete chloroplast genome of A. hypogaea $\mathrm{L}$. will be an invaluable resource for designing and evaluating efficient chloroplast transformation experiments. 


\section{MATERIALS AND METHODS}

\section{Plant Material and Genome Sequencing}

The seeds of A. hypogaea L. Co7 variety were obtained from Tamilnadu Agricultural University, Coimbatore, India. The plants were grown in the green house facility at SRM University, Kattankulathur, India. Leaves from 1-month old plant were used for total genomic DNA isolation using DNeasy Plant Mini Kit (Qiagen, Germany). A paired-end library with an average insert size of about $400 \mathrm{bp}$ was constructed as per the manufacturer's protocol (Illumina Inc., USA). The library quality was assessed on CaliperLabChip GX using High Sensitivity Assay Kit (Caliber, USA). It was then hybridized on a flow cell for generating clonal clusters on cBOT using Truseq PE Cluster Kit v3-cBot-HS (Illumina Inc., USA). Sequencing by synthesis was performed on Illumina Hiseq 2500 using Truseq v3-HS kit to generate 100 bp paired end reads (Illumina Inc., USA).

\section{Genome Assembly and Validation}

The per base quality of the raw paired-end reads $(51,650,486)$ of $100 \mathrm{bp}$ was assessed by FastQC v0.11.2 (Andrews, 2010). The adapter trimming and quality filtering was done using Cutadapt v1.7.1 (Martin, 2011) and Sickle v1.33 (Joshi and Fass, 2011) tools respectively. A phred score of 20 was used for quality filtering. The quality filtered paired-end reads $(49,299,308)$ were subjected to de novo assembly using three different de novo assemblers such as Velvet v1.2.10 (Zerbino and Birney, 2008), SOAPdenovo v2.04 (Luo et al., 2012) and Edena v3.131028 (Hernandez et al., 2008). The assembled contigs were pooled and ordered against the complete CP genome of closest relative Acacia ligulata L. as the reference using Mauve v2.3.1 tool (Darling et al., 2010; Williams et al., 2015). The gaps in the genome were filled by manual alignment of paired-end reads using overlapping method (Natarajan and Parani, 2015) and primer walking (Sanger sequencing method). Validation of the junctions between the single copy regions and the inverted repeats was done by Sanger sequencing using specific primers. The filtered reads were mapped against the assembled CP genome of A. hypogaea L. to calculate the genome coverage. The complete $\mathrm{CP}$ genome of A. hypogaea L. was annotated using DOGMA (Wyman et al., 2004).

\section{RESULTS AND DISCUSSION}

The size of the complete CP genome of A. hypogaea L. was found to be $156,391 \mathrm{bp}$. The genome coverage was calculated to be $2122 \mathrm{x}$ with $3,863,475$ quality filtered reads mapped to the assembled CP genome. The CP genome exhibited a quadripartite structure consisting of LSC and SSC regions of 85,946 bp and 18,797 bp respectively, with a pair of inverted repeats (IRa and IRb) of $25,824 \mathrm{bp}$ each separating them. The overall GC content of the complete chloroplast genome was $36.4 \%$ and the individual GC content for LSC, SSC, and IRs was 33.8\%, 30.2\%, and $42.8 \%$ respectively. A total of 110 genes were annotated including 76 protein coding genes, 30 tRNA genes, and 4 rRNA genes. Six of the protein coding genes and the 3' exon of rps12 are duplicated in the IR regions. Six of the tRNA genes and four of the rRNA genes are also duplicated in the IR regions. The presence of one or two introns were identified in the 13 genes, which includes 8 protein coding genes and 5 tRNAgenes (Table 1). The complete CP genome sequence of A. hypogaea that is reported here for the first time will be an invaluable resource for designing and evaluating efficient chloroplast transformation experiments and to improve the desired traits.

\section{DEPOSITED DATA AND INFORMATION TO THE USER}

The complete data from the current study was submitted at NCBI under the BioProject ID PRJNA314013 and BioSample ID SAMN04527043. The assembled complete chloroplast genome sequence was submitted to NCBI Genbank with an accession number KX257487 (http://www.ncbi.nlm.nih.gov/nuccore/KX257487). The raw reads in compressed FASTQ were submitted to SRA database at NCBI under the accession number SRP076091 (http://www.ncbi.nlm.nih.gov/sra/SRP076091). Users can download and reuse the data for research purpose only with an acknowledgement to us and quoting this paper as reference to the data.

TABLE 1 | List of genes found in the A. hypogaea L. chloroplast genome.

\begin{tabular}{|c|c|c|}
\hline S.No & Group of genes & Gene names \\
\hline 1 & ATP synthase & $\operatorname{atp} A, \operatorname{atp} B, \operatorname{atp} E, \operatorname{atp} F^{*}, \operatorname{atpH}$, atpl \\
\hline 2 & Cytochrome b/f complex & pet $A$, pet $B$, petD, petG, petL, pet $N$ \\
\hline 3 & $\mathrm{NADH}$ dehydrogenase & $\begin{array}{l}n d h A^{*}, n d h C, n d h D, n d h E, n d h F \\
n d h G, n d h H, n d h l, n d h J\end{array}$ \\
\hline 4 & Photosystem I & psaA, psaB, psaC, psal, psaJ \\
\hline 5 & Photosystem II & $\begin{array}{l}p s b A, p s b B, p s b C, p s b D, p s b E, p s b F \\
p s b H, p s b l, p s b J, p s b K, p s b L, p s b M, \\
p s b N, p s b T, p s b Z\end{array}$ \\
\hline 6 & Proteins of unknown function & $y c f 1, y c f 2, y c f 3^{\star \star}, y c f 4$, orf42, $y c f 68^{\star}$ \\
\hline 7 & Ribosomal proteins (SSU) & $\begin{array}{l}r p s 2, r p s 3, r p s 4, r p s 7, r p s 8, r p s 11 \\
r p s 12^{\#}, r p s 14, r p s 15, r p s 18, r p s 19\end{array}$ \\
\hline 8 & Ribosomal proteins (LSU) & $\begin{array}{l}r p / 2^{*}, r p / 14, \text { rp/16, rp/20, rp/23, rp/32, } \\
\text { rp/33, rp/36 }\end{array}$ \\
\hline 9 & Ribosomal RNAs & $r r n 4.5, r r n 5, r r n 16, r r n 23$ \\
\hline 10 & RNA polymerase & rpoA, rpoB, rpoC1*, rpoC2 \\
\hline 11 & Other genes & $a c c D, c c s A, c e m A, c / p P^{\star \star}$, matK, $r b c L$ \\
\hline 12 & Transfer RNAs & 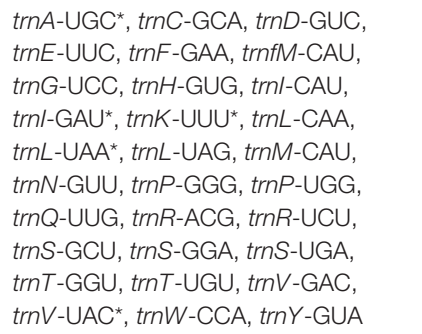 \\
\hline
\end{tabular}

${ }^{*}$ Contains one intron ${ }^{*}$ Contains two introns ${ }^{\#}$ Exhibits trans-splicing. 


\section{AUTHOR CONTRIBUTIONS}

PN conceived the study and acquired the funding; SKP and SP performed the genome assembly and analysis; SKP, PN, and PM drafted the manuscript. All authors approved the final manuscript.

\section{FUNDING}

The project was funded by Department of Biotechnology (DBT), Government of India, under the Rapid Grant for

\section{REFERENCES}

Andrews, S. (2010). FastQC: a Quality Control Tool for High Throughput Sequence Data. Available online at: http://www.bioinformatics.babraham.ac.uk/projects/ fastqc

Daniell, H., Kumar, S., and Dufourmantel, N. (2005). Breakthrough in chloroplast genetic engineering of agronomically important crops. Trends Biotechnol. 23, 238-245. doi: 10.1016/j.tibtech.2005.03.008

Darling, A. E., Mau, B., and Perna, N. T. (2010). progressiveMauve: multiple genome alignment with gene gain, loss and rearrangement. PLoS ONE 5:e11147. doi: 10.1371/journal.pone.0011147

David, J. B., Steven, B. C., Lutz, F., Guodong, H., Andrew, D. F., Ethalinda, K. S. C., et al. (2016). The genome sequence of Arachis duranensis and Arachis ipaensis, the diploid ancestors of cultivated peanut. Nat. Genet. 48, 438-446. doi: $10.1038 /$ ng. 3517

FAOSTAT (2015). Production Statistics for Groundnut. Available online at: http://faostat3.fao.org/ (accessed 18.05.2016).

Hernandez, D., François, P., Farinelli, L., and Østerås, M., Schrenzel, J. (2008). De novo bacterial genome sequencing: millions of very short reads assembled on a desktop computer. Genome Res. 18, 802-809. doi: 10.1101/gr.072033.107

Joshi, N. A., and Fass, J. N. (2011). Sickle: a Sliding-Window, Adaptive, QualityBased Trimming Tool for FastQ Files (Version 1.33) [Software]. Available online at: https://github.com/najoshi/sickle

Kochert, G., Stalker, H. T., Gimenes, M., Galgaro, L., Catalina, R. L., and Moore, K. (1996). RFLP and cytogenetic evidence on the origin and evolution of allotetraploid domesticated peanut, Arachis hypogaea (Leguminosae) Am. J. Bot. 83, 1282-1291. doi: 10.2307/2446112

Luo, R., Liu, B., Xie, Y., Li, Z., Huang, W., Yuan, J., et al. (2012). SOAPdenovo2: an empirically improved memory-efficient short-read de novo assembler. GigaScience 1:18. doi: 10.1186/2047-217X-1-18

Martin, M. (2011). Cutadapt removes adapter sequences from high-throughput sequencing reads. EMBnet J. 17, 10-12. doi: 10.14806/ej.17.1.200

Natarajan, P., and Parani, M. (2015). First complete genome sequence of a probiotic Enterococcus faecium strain T-110 and its comparativegenome
Young Investigator (RGYI) scheme (BT/PR6394/GBD/27/422/ 2012).

\section{ACKNOWLEDGMENTS}

This project was supported by Department of Biotechnology (DBT), Government of India (BT/PR6394/GBD/27/422/2012). The High Performance Cluster Computing Facility at SRM University was used for the genome assembly and analysis.

analysis with pathogenic and non-pathogenic Enterococcus faecium genomes. J. Genet. Genomics 42, 43-46. doi: 10.1016/j.jgg.2014.07.002

Ravi, V., Khurana, J., Tyagi, A., and Khurana, P. (2008). An update on chloroplast genomes. Plant Syst. Evol. 271. 101-122. doi: 10.1007/s00606-007-0608-0

Raubeson, L. A., and Jansen, R. K. (2005). “Chloroplast genomes of plants," in Plant Diversity and Evolution: Genotypic and Phenotypic Variation in Higher Plants, ed R. J. Henry (Wallingford, CT: CABI), 45-68.

Shiv, K. B. (1982). Fatty acid composition of 16 groundnut (Arachis hypogaea L.) cultivars grown under Malaysian conditions. Pertanika 5, 20-24.

Williams, A. V., Boykin, L. M., Howell, K. A., Nevill, P. G., and Small, I. (2015). The complete sequence of the Acacia ligulata chloroplast genome reveals a highly divergent clpP1 gene. PLoS ONE 10:e0125768. doi: 10.1371/journal.pone.0125768

Wu, Z. Q., and Ge, S. (2012). Phylogeny of the BEP clade in grasses revisited: evidence from whole genome sequences of chloroplast. Mol. Phylogenet. Evol. 62, 578-578. doi: 10.1016/j.ympev.2011.10.019

Wyman, S. K., Jansen, R. K., and Boore, J. L. (2004). Automatic annotation of organellar genomes with DOGMA. Bioinformatics 20, 3252-3255. doi: 10.1093/bioinformatics/bth352

Zerbino, D. R., and Birney, E. (2008). Velvet: algorithms for de novo short read assembly using de Bruijn graphs. Genome Res. 18, 821-829. doi: 10.1101/gr.074492.107

Conflict of Interest Statement: The authors declare that the research was conducted in the absence of any commercial or financial relationships that could be construed as a potential conflict of interest.

Copyright (C) 2016 Prabhudas, Prayaga, Madasamy and Natarajan. This is an openaccess article distributed under the terms of the Creative Commons Attribution License (CC BY). The use, distribution or reproduction in other forums is permitted, provided the original author(s) or licensor are credited and that the original publication in this journal is cited, in accordance with accepted academic practice. No use, distribution or reproduction is permitted which does not comply with these terms. 